\title{
Flows on Classes of Regular Semigroups and Cauchy Categories
}

\author{
Suha Ahmed Wazzan (iD \\ Department of Mathematics, Science Faculty, King Abdulaziz University, Girls Campus, 21589 Jeddah, Saudi Arabia \\ Correspondence should be addressed to Suha Ahmed Wazzan; swazzan@kau.edu.sa
}

Received 4 February 2019; Accepted 3 April 2019; Published 15 April 2019

Academic Editor: Radomír Halaš

Copyright (C) 2019 Suha Ahmed Wazzan. This is an open access article distributed under the Creative Commons Attribution License, which permits unrestricted use, distribution, and reproduction in any medium, provided the original work is properly cited.

We consider the structure of the flow monoid for some classes of regular semigroups (which are special case of flows on categories) and for Cauchy categories. In detail, we characterize flows for Rees matrix semigroups, rectangular bands, and full transformation semigroups and also describe the Cauchy categories for some classes of regular semigroups such as completely simple semigroups, Brandt semigroups, and rectangular bands. In fact, we obtain a general structure for the flow monoids on Cauchy categories.

\section{Introduction and Preliminaries}

The term flow monoid first arose in unpublished typescript of Chase; in this manuscript, Chase determines the structure of the flow monoid (named it in his paper incidence monoid) and its group of units in general category theory which has categories with fixed vertices set $X$. In [1], Gilbert adopted the term flow monoid which connects the concepts with vector fields. Gilbert was solely interested in studying flow monoid in the theory of regular semigroup. He depicted the structure of the flow monoid in accordance to the Green relations on the semigroup. The aim of this paper is to describe flow monoids for some classes of regular semigroups. We characterize flows for Rees matrix semigroups, rectangular bands, and full transformation semigroups. We describe the Cauchy categories for some classes of regular semigroups such as completely simple semigroups, Brandt semigroups, and rectangular bands. We find the general structure of the flow monoids on Cauchy categories.

A directed graph consists of two sets: a vertex set and an arrow set and two mappings: dom $: E \longrightarrow V$ and the codomain operation cod $: E \longrightarrow V$. There are many possible formulations of the definition of categories (see, for instance, [2-4]). As in [5], we shall regard a category as a directed graph with extra structure in the mean of abstract algebra. We start with a directed graph with the term objects used for the vertices, and the term arrows used for directed edges, and which has supplementary operations:

(C1) Identity, which appoints to each object $a$ an arrow $i d_{a}=1_{a}: a \longmapsto a$;
(C2) Composition, which designates each two arrows $f$ and $g$ satisfying domg $=\operatorname{codf}$ with an arrow $g f$ : $\operatorname{domf} \longmapsto \operatorname{codg}$. Composition satisfies two conditions:

(i) Associativity: for objects and arrows such that $a \longmapsto{ }^{f} b \longmapsto^{g} c \longmapsto^{k} d$, we have the equality

$$
k(g f)=(k g) f .
$$

(ii) Identity: for each arrow $f: a \longmapsto b$, we have the composition with identity arrows $1_{a}$ and $1_{b}$ : $f 1_{a}=f$ and $1_{b} f=f$.

In general, a category contains objects set $X$ and arrows set $A$ between objects; arrows can sometimes be composed, whenever they fit together with source and target maps $\sigma, \tau$ : $A \longrightarrow X$, and an identity map $e: X \longrightarrow A$ such that $(x e) \sigma=$ $x=(x e) \tau$. Whenever $a \tau=b \sigma$, every object has an identity arrow and so the composition of arrows is associative.

According to [1], a flow is a set-theoratic section to the source map. A flow on a category $\mathbb{C}$ with vertex set $X$ is a function $\varphi: X \longrightarrow \mathbb{C}$ that is a section to the source map: that is, for all $x \in X,(x \varphi) \sigma=x$, let $\Phi(\mathbb{C})$ denote the set of all flows on $\mathbb{C}$; then $\Phi(\mathbb{C})$ is a monoid, with composition * defined by

$$
x(\varphi * \psi)=x \varphi((x \varphi \tau)) \psi
$$

and identity $e: x \longmapsto x$. 
In Section 3, we characterize the structure of the flow monoids for some regular semigroups such as full transformation semigroups, Rees matrix semigroups, and rectangular band.

Cauchy category of a semigroup $S$ is a small category whose vertices set is the set $E(S)$ of idempotents of $S$. An arrow from $f$ to $e$ is a triple $(e, s, f)$ where $s \in e S f$. Composition of arrows is given by

$$
(e, s, f)(f, t, g)=(e, s t, g) \text {. }
$$

The identity at $e$ is $(e, e, e)$ by [6]. Moreover, again by the same reference, the Cauchy category known as Karoubi envlope $\mathrm{k}(S)$ (or Cauchy completion or idempotent splitting) is a full subcategory of $\mathbb{C}(S)$ which is named as the Schützenberger category of $S$ whose vertices are elements of the semigroup $S$. The Schützenberger category $\mathbb{C}(S)$ of a semigroup $S$ is defined as follows. The vertices set of $\mathbb{C}(S)$ is $S$. Also an arrow from $t$ to $s$ is a triple $(s, u, t)$ such that $u \in s S^{1} \cap S^{1} s$. The composition of arrows is given by

$$
(s, x t, t)(t, t y, r)=(s, x t y, r),
$$

where $x, y \in S^{1}$ and the identity at $s$ is $(s, s, s)$.

In Section 4, we describe Cauchy categories for some small regular semigroups such as rectangular bands, Brandt semigroups, and completely simple semigroups and then find the general structure for the flow monoid on Cauchy categories. We actually prove that, in the Cauchy category $\varrho(S)$, we have exactly one arrow from $e$ to $f$ (for all $e, f \in$ $E(S))$ if and only if $S$ is a rectangular band.

\section{Flows on Regular Semigroups}

Let $S$ be a regular semigroup, so for all $a \in S$ there exists $b \in S$ such that $a b a=a$ and $b a b=b$. We call $b$ an inverse for $a$. An element $a$ may have many inverses, and the set of inverses of $a$ is denoted by $V(a)$. At the same time, an idempotent in $S$ is an element $e \in S$ such that $e^{2}=e$. If $b \in V(a)$, then $a b$ and $b a$ are idempotents. Let $E(S)$ be the set of idempotents. In regular semigroup, $E(S)$ need not be a subsemigroup since the product of two idempotents need not be an idempotent element. If $S$ is regular and $E(S)$ is a subsemigroup of $S$, then $S$ is called orthodox (cf. [7]).

A flow $\alpha$ on $S$ is a pair of functions $\alpha_{1}, \alpha_{2}: E(S) \longrightarrow S$ with the properties

$$
\text { for all } e \in E(S) \text {, }
$$

$$
\text { the elements } \alpha_{2}(e) \in V\left(\alpha_{1}(e)\right) \text {; }
$$

and

$$
e=\alpha_{1}(e) \alpha_{2}(e) .
$$

Due to [1], by letting $\Phi(S)$ as the set of all flows on $S$, one can compose flows in the following way: if $\alpha$ and $\beta$ are flows on $S$, then a pair of mappings $\gamma_{1}, \gamma_{2}: E(S) \longrightarrow S$ is defined as follows:

$$
\begin{gathered}
\gamma_{1}(e)=\alpha_{1}(e) \beta_{1}\left(\alpha_{2}(e) \alpha_{1}(e)\right) \\
\text { and } \gamma_{2}(e)=\beta_{2}(e)\left(\alpha_{2}(e) \alpha_{1}(e)\right) \alpha_{2}(e) .
\end{gathered}
$$

Thus we have the following first lemma of this section.
Lemma 1. The pair of mappings $\left(\gamma_{1}, \gamma_{2}\right)$ is a flow on $S$.

Proof. For all $e \in E(S)$ and $\gamma_{2}(e) \in V\left(\gamma_{1}(e)\right)$, we have to prove that

$$
\begin{aligned}
\gamma_{1}(e) \gamma_{2}(e) \gamma_{1}(e) & =\gamma_{1}(e) \\
\text { and } \gamma_{2}(e) \gamma_{1}(e) \gamma_{2}(e) & =\gamma_{2}(e) .
\end{aligned}
$$

Now

$$
\begin{aligned}
\gamma_{1}(e) \gamma_{2}(e) \gamma_{1}(e)=\alpha_{1}(e) \\
\cdot \underbrace{\beta_{1}\left(\alpha_{2}(e) \alpha_{1}(e)\right) \beta_{2}(e)\left(\alpha_{2}(e) \alpha_{1}(e)\right)} \alpha_{2}(e) \alpha_{1}(e) \\
\cdot \beta_{1}\left(\alpha_{2}(e) \alpha_{1}(e)\right) \\
=\underbrace{\alpha_{1}(e) \alpha_{2}(e) \alpha_{1}(e) \alpha_{2}(e) \alpha_{1}(e) \beta_{1}\left(\alpha_{2}(e) \alpha_{1}(e)\right)}
\end{aligned}
$$$$
[\beta \text { is a flow on } S]
$$

$$
=\underbrace{\alpha_{1}(e) \alpha_{2}(e) \alpha_{1}(e)} \beta_{1}\left(\alpha_{2}(e) \alpha_{1}(e)\right)
$$

[ $\alpha$ is a flow on $S]$

$=\alpha_{1}(e) \beta_{1}\left(\alpha_{2}(e) \alpha_{1}(e)\right) \quad[\alpha$ is a flow on $S]$

$=\gamma_{1}(e)$.

Similarly, we obtain $\gamma_{2}(e) \gamma_{1}(e) \gamma_{2}(e)=\gamma_{2}(e)$. Additionally, we also have

$$
\begin{aligned}
\gamma_{1} & (e) \gamma_{2}(e) \\
= & \alpha_{1}(e) \underbrace{\beta_{1}\left(\alpha_{2}(e) \alpha_{1}(e)\right) \beta_{2}(e)\left(\alpha_{2}(e) \alpha_{1}(e)\right)} \alpha_{2}(e) \\
= & \underbrace{\alpha_{1}(e) \alpha_{2}(e) \alpha_{1}(e) \alpha_{2}(e)} \quad[\beta \text { is a flow on } S] \\
= & e \cdot e=e^{2}=e \quad[\alpha \text { is a flow on } S] .
\end{aligned}
$$

Hence the pair of mappings $\left(\gamma_{1}, \gamma_{2}\right)$ is a flow on $S$.

Define the flow $\gamma=\left(\gamma_{1}, \gamma_{2}\right)$ to be the product of the flows $\alpha=\left(\alpha_{1}, \alpha_{2}\right)$ and $\beta=\left(\beta_{1}, \beta_{2}\right)$ in $\Phi(S)$, and write $\gamma=\alpha * \beta$. Thus we get the following proposition.

Proposition 2. $(\Phi(S), *)$ is a monoid which is called the flow monoid of $S$.

Proof. To show that $\Phi(S)$ is a semigroup, we first need to prove the operation $*$ is associative. Let $a, b, c$ be flows on $S$, and so each of those is a pair of mapping from $E(S)$ to $S$. To prove $(a * b) * c=a *(b * c)$, let $p=(a * b) * c$ and $q=a *(b * c)$. We then have pairs of maps $p_{1}, p_{2}$ and $q_{1}, q_{2}$ again from $E(S)$ to $S$. So we have to show $p_{1}=q_{1}$ and $p_{2}=q_{2}$. Now

$$
\begin{aligned}
& p_{1}(e)=(a * b)_{1}(e) c_{1}\left((a * b)_{2}(e)(a * b)_{1}(e)\right) \\
& =a_{1}(e) b_{1}\left(a_{2}(e) a_{1}(e)\right) c_{1}\left(b_{2}\left(a_{2}(e) a_{1}(e)\right) a_{2}(e)\right. \\
& \cdot a_{1}(e) b_{1}\left(a_{2}(e) a_{1}(e)\right)
\end{aligned}
$$


Since $p$ is a flow, then $a_{2}(e) a_{1}(e)=b_{1}\left(a_{2}(e) a_{1}(e)\right) b_{2}\left(a_{2}(e) a_{1}(e)\right)$, and so in $p_{1}$ we have

$$
\begin{aligned}
c_{1}( & \left.b_{2}\left(a_{2}(e) a_{1}(e)\right) a_{2}(e)\right) a_{1}(e) b_{1}\left(a_{2}(e) a_{1}(e)\right) \\
= & c_{1}\left(b_{2}\left(a_{2}(e) a_{1}(e)\right) b_{1}\left(a_{2}(e) a_{1}(e)\right)\right. \\
\cdot & \left.b_{2}\left(a_{2}(e) a_{1}(e)\right) b_{1}\left(a_{2}(e) a_{1}(e)\right)\right)=c_{1}\left(b _ { 2 } \left(a_{2}(e)\right.\right. \\
\cdot & \left.a_{1}(e)\right) b_{1}\left(a_{2}(e) a_{1}(e)\right)
\end{aligned}
$$

[since $p_{1}(f)$ is the inverse for $p_{2}(f)$ for all $f \in S$ ] which implies that

$$
\begin{aligned}
p_{1}(e)= & a_{1}(e) b_{1}\left(a_{2}(e) a_{1}(e)\right) \\
& \cdot c_{1}\left(b_{2}\left(a_{2}(e) a_{1}(e)\right) b_{1}\left(a_{2}(e) a_{1}(e)\right)\right)
\end{aligned}
$$

whereas $q_{1}(e)=a_{1}(e)(b * c)_{1}\left(a_{2}(e) a_{1}(e)\right)$. Thus

$$
\begin{aligned}
q_{1}(e)= & a_{1}(e) b_{1}\left(a_{2}(e) a_{1}(e)\right) \\
& \cdot c_{1}\left(b_{2}\left(a_{2}(e) a_{1}(e)\right) b_{1}\left(a_{2}(e) a_{1}(e)\right)\right) .
\end{aligned}
$$

Therefore (13) and (14) together imply that $p_{1}=q_{1}$. Similarly, we get $p_{2}=q_{2}$, and hence $*$ is an associative operation. Now the trivial flow $\varepsilon$ on $S$ is the pair of mappings ( $i d, i d)$, where $i d$ is the identity map on $E(S)$. So, for all $e \in E(S)$, we have $\varepsilon_{i}(e)=e$, where $i=1,2$. Then $\epsilon$ is the identity element in $\Phi(S)$ since

$$
\varepsilon=(i d, i d): E(S) \longrightarrow S
$$

$$
\text { such that } \varepsilon_{1}(e)=e, \varepsilon_{2}(e)=e, \forall e \in E(S) \text {. }
$$

As the next step, for all $a \in \Phi(S)$, we have to show $\varepsilon * a=$ $a * \varepsilon=a$, where $a$ is a flow on $S$. In other words, $(\varepsilon * a)_{1}=a_{1}$ and $(\varepsilon * a)_{2}=a_{2}$. Now, from the equality $(\varepsilon * a)_{1}=p_{1}$, we get

$$
\begin{aligned}
p_{1}(e) & =\varepsilon_{1}(e) a_{1}\left(\varepsilon_{2}(e) \varepsilon_{1}(e)\right)=e a_{1}(e \cdot e)=e a_{1}\left(e^{2}\right) \\
& =e a_{1}(e) \\
& =a_{1}(e) a_{2}(e) a_{1}(e) \quad \text { by }(6) \\
& =a_{1}(e) \quad \text { by }(5) .
\end{aligned}
$$

Hence we get $p_{1}(e)=a_{1}(e)$ which implies that $(\varepsilon * a)_{1}=$ $a_{1}$. With a similar calculation, from the equation $(\varepsilon * a)_{2}=p_{2}$, we obtain $p_{2}(e)=a_{2}(e)$ which gives $(\varepsilon * a)_{2}=a_{2}$.

Therefore $\varepsilon$ is the identity element in $\Phi(S)$, and so it becomes a monoid.

\section{The Structure of Flow Monoids on Classes of Regular Semigroups}

In this section, by describing the construction of the flow monoid on a regular semigroup (which is a special case of a flow on a category), we will investigate the special classes of regular semigroups and flow monoids more precisely.

In the following example, by taking into account a regular semigroup $S=7_{X}$ where $X=\{1,2\}$ (in fact, by [8], $S$ is the full transformation semigroup on $X)$, we will compute the number of elements in the flow monoid $\Phi(S)$.
TABLE 1: Cayley table of $\neg\{1,2\}$.

\begin{tabular}{lllll}
\hline$\neg_{\{1,2\}}$ & 1 & $\tau$ & $e_{1}$ & $e_{2}$ \\
\hline 1 & 1 & $\tau$ & $e_{1}$ & $e_{2}$ \\
\hline$\tau$ & $\tau$ & 1 & $e_{2}$ & $e_{1}$ \\
\hline$e_{1}$ & $e_{1}$ & $e_{1}$ & $e_{1}$ & $e_{1}$ \\
\hline$e_{2}$ & $e_{2}$ & $e_{2}$ & $e_{2}$ & $e_{2}$ \\
\hline
\end{tabular}

Example 1. Recall that the full transformation semigroup $\urcorner_{X}$ on a set $X$ consists of all mappings from $X$ to itself under the operation composition of mappings. It is known that $|\neg(x)|=$ $n^{n}$ when $|X|=n$.

By taking any element $f \in \neg(x)$, we will first show that $\neg(x)$ is actually a regular semigroup. To do that we need to find a $g \in \neg(x)$ such that $f=f g f$ and $g=g f g$. Since $f$ is a map from $X$ to itself, we can define it as $f\left(a_{y}\right)=y$ where $y \in \operatorname{im}(f)$ and $a_{y} \in X$. Also define $g: X \longrightarrow X$ by

$$
g(y)= \begin{cases}a_{y} & \text { if } y \in \operatorname{im}(f) \\ y & \text { if } y \notin \operatorname{im}(f)\end{cases}
$$

Then

$$
\begin{aligned}
f g f(x) & =f g(y) \quad \text { if } y=f(x) \\
& =f\left(a_{y}\right)=y=f(x)
\end{aligned}
$$

which implies $f g f=f$. Similarly we can get $g f g=g$. Hence $\neg(x)$ is a regular semigroup.

Now we compute the number of elements in the flow monoid $\Phi\left(\neg_{\{1,2\}}\right)$. In fact, $\urcorner_{\{1,2\}}$ is defined by

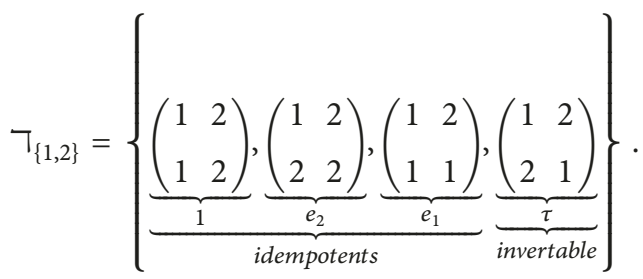

Recall that $a \in V(b) \Longleftrightarrow a b a=a, b a b=b$. In Table 1, we find all inverses.

It is clear from the table that $V(1)=\{1\}, V(\tau)=\{\tau\}$, and $V\left(e_{1}\right)=V\left(e_{2}\right)=\left\{e_{1}, e_{1}\right\}$. Now, let

$$
\begin{aligned}
&\left.\varphi_{i}: E(\urcorner_{\{1,2\}}\right)\left.\longrightarrow(\urcorner_{\{1,2\}}\right), \quad i=1,2 \\
& \varphi\left.=\left(\varphi_{1}, \varphi_{2}\right) \in \Phi(\urcorner_{\{1,2\}}\right) \\
& \text { if } \varphi_{1}(x) \varphi_{2}(x)=x \text { and } \varphi_{2}(x) \in V\left(\varphi_{1}(x)\right)
\end{aligned}
$$

We can get a (bad) estimate of the size of $\Phi(S)$ here: number of maps $E(S) \longrightarrow S \times S$ without any restriction is $16^{3}$-much too big. Let us look in term at the possibilities for $\varphi_{1}, \varphi_{2}: E(S) \longrightarrow S$ for which element of $E(S)$.

For $x=1$, we have two possibilities (1) $\varphi_{1}(x)=\varphi_{2}(x)=1$ or (2) $\varphi_{1}(x)=\varphi_{2}(x)=\tau$. 
TABLE 2: Flows on $7\{1,2\}$.

\begin{tabular}{ccccccccc}
\hline & $\phi$ & $\beta$ & $\gamma$ & $\sigma$ & $\psi$ & $\tau$ & $\delta$ & $\alpha$ \\
\hline 1 & $(1)$ & $(1)$ & $(1)$ & $(1)$ & $(2)$ & $(2)$ & $(2)$ & $(2)$ \\
\hline$e_{1}$ & $(3)$ & $(4)$ & $(3)$ & $(4)$ & $(3)$ & $(4)$ & $(3)$ & $(4)$ \\
\hline$e_{2}$ & $(5)$ & $(5)$ & $(6)$ & $(6)$ & $(5)$ & $(5)$ & $(6)$ & $(6)$ \\
\hline
\end{tabular}

For $x=e_{1}$, we have six possibilities for choosing two elements in $S$ whose product is $e_{1}$ :

$$
\begin{aligned}
\varphi_{1}(x) & =1, \\
\varphi_{2}(x) & =e_{1} \\
\varphi_{1}(x) & =\tau, \\
\varphi_{2}(x) & =e_{2} \\
\varphi_{1}(x) & =e_{1}, \\
\varphi_{2}(x) & =1 \\
\varphi_{1}(x) & =e_{1}, \\
\varphi_{2}(x) & =\tau \\
\text { (3) } \varphi_{1}(x) & =e_{1}, \\
\varphi_{2}(x) & =e_{1} \\
\varphi_{1}(x) & =e_{1}, \\
\varphi_{2}(x) & =e_{2}
\end{aligned}
$$

For $x=e_{2}$, as for $e_{1}$, we can only choose

$$
\text { (5) } \begin{aligned}
\varphi_{1}(x) & =e_{2}, \\
\varphi_{2}(x) & =e_{2} \\
\text { (6) } \varphi_{1}(x) & =e_{2}, \\
\varphi_{2}(x) & =e_{1}
\end{aligned}
$$

Therefore, we get eight flows on $S$ as shown in Table 2 .

A sample of the composition can be considered as $\varphi, \psi \in$ $\Phi(S), \varphi_{1}(1)=\tau=\varphi_{2}(1), \varphi_{1}\left(e_{1}\right)=e_{1}, \varphi_{2}\left(e_{1}\right)=e_{2}, \varphi_{1}\left(e_{2}\right)=$ $e_{2}=\varphi_{2}\left(e_{2}\right)$, and $\psi_{1}(1)=\tau=\psi_{2}(1), \psi_{1}\left(e_{1}\right)=e_{1}, \psi_{2}\left(e_{1}\right)=$ $e_{2}, \psi_{1}\left(e_{2}\right)=e_{2}=\psi_{2}\left(e_{2}\right)$. Then $\varphi * \psi \in \Phi(S)$ satisfies

$$
\begin{aligned}
& (\varphi * \psi)_{1}(x)=\varphi_{1}(x) \psi_{1}\left(\varphi_{2}(x) \varphi_{1}(x)\right), \\
& (\varphi * \psi)_{2}(x)=\psi_{2}\left(\varphi_{2}(x) \varphi_{1}(x)\right) \varphi_{2}(x) .
\end{aligned}
$$

For the cases

$$
\begin{aligned}
& x=1, \text { we have }(\varphi * \psi)_{1}(1)=\tau \psi_{1}(1)=\tau^{2}=1 \text { and } \\
& (\varphi * \psi)_{2}(x)=1\left(\text { same as }(\varphi * \psi)_{1}\right) \\
& x=e_{1} \text {, we have }(\varphi * \psi)_{1}\left(e_{1}\right)=e_{1} \psi_{1}\left(e_{2} e_{1}\right)=e_{1} \psi_{1}\left(e_{2}\right)= \\
& e_{1} e_{2}=e_{1} \text { and } \\
& (\varphi * \psi)_{2}\left(e_{1}\right)=\psi_{2}\left(e_{2} e_{1}\right) \varphi_{2}\left(e_{1}\right)=\psi_{2}\left(e_{2}\right) \varphi_{2}\left(e_{1}\right) \\
& =e_{1} e_{2}=e_{1}
\end{aligned}
$$

$x=e_{2}$, we have $(\varphi * \psi)_{1}\left(e_{2}\right)=e_{2} \psi_{1}\left(e_{2}\right)=e_{2} e_{2}=e_{2}$ and

$$
(\varphi * \psi)_{2}\left(e_{2}\right)=\psi_{2}\left(e_{2}\right) \varphi_{2}\left(e_{2}\right)=e_{1} e_{2}=e_{1}
$$

To understand the full structure, we would need to complete the $8 \times 8$ table for the composition in $\Phi(S)$. Note that we have the trivial flow $\varepsilon, \varepsilon_{1}(x)=x, \varepsilon_{2}(x)=x$, and $\varphi * \varepsilon=\varepsilon * \varphi=\varphi$ for all $\varphi \in \Phi(S)$.

In [1, Proposition 3.3], Gilbert characterized that a simplicity groupoid arises from the Nambooripad construction. In the following example, we will investigate the flow monoid on a rectangular band.

Example 2. Recall that a semigroup $S$ is a band if every element of $S$ is an idempotent and a rectangular band if $a b a=$ $a$ for all $a, b$ in $S$. Define $(a, b)(c, d)=(a, d) \in X \times X=S$. Then $S$ is regular and for all $(a, b) \in S, V(a, b)=S$, and $E(S)=S$. For flow on $S$, we have

$$
\begin{aligned}
\varphi_{1}(x) \varphi_{2}(x) & =x \\
\text { and } \varphi_{2}(x) & \in V\left(\varphi_{1}(x)\right)
\end{aligned}
$$

which is trivially true. So, for a flow is now a pair of maps $[i=1,2] \varphi_{i}: S \longrightarrow S$ such that $\varphi_{1}(x) \varphi_{2}(x)=x$, let us suppose

$$
\begin{aligned}
& \varphi_{1}(a, b)=\left(\varphi_{11}(a, b), \varphi_{12}(a, b)\right) \in S \\
& \varphi_{2}(a, b)=\left(\varphi_{21}(a, b), \varphi_{22}(a, b)\right) \in S .
\end{aligned}
$$

So $\varphi_{1}(a, b) \varphi_{2}(a, b)=\left(\varphi_{11}(a, b), \varphi_{22}(a, b)\right)=(a, b)$ which gives $\varphi_{11}(a, b)=a, \varphi_{22}(a, b)=b$. Therefore

$$
\begin{aligned}
\varphi_{1}(a, b) & =\left(a, \varphi_{12}(a, b)\right) \\
\text { and } \varphi_{2}(a, b) & =\left(\varphi_{21}(a, b), b\right),
\end{aligned}
$$

where $\varphi_{12}, \varphi_{21}: X \times X \longrightarrow X$. So we get a flow whenever we choose any pair of mappings $f, g: X \longrightarrow X$ and the flow is given by $\varphi_{1}(a, b)=(a, f(a, b))$ and $\varphi_{2}(a, b)=(g(a, b), b)$, or could combine $f, g$ into single mappings $X \times X \longrightarrow X \times X$ and so a mapping $S \longrightarrow S$. Now suppose pairs of functions $(f, g): X \times X \longrightarrow X$ determine the flow $\varphi$ a pair of functions $\varphi_{i}: S \longrightarrow S$, for $i=1,2$, such that

$$
\begin{aligned}
\varphi_{1}(a, b) & =(a, f(a, b)) \\
\text { and } \varphi_{2}(a, b) & =(g(a, b), b)
\end{aligned}
$$

and $(h, k): X \times X \longrightarrow X$ determines the flow $\psi$ a pair of functions $\psi_{i}: S \longrightarrow S$, for $i=1,2$, such that

$$
\begin{aligned}
\psi_{1}(a, b) & =(a, h(a, b)) \\
\text { and } \psi_{2}(a, b) & =(k(a, b), b) .
\end{aligned}
$$


Then the corresponding functions $\varphi * \psi: X \times X \longrightarrow X$ are defined by

$$
\begin{aligned}
(\varphi & * \psi)_{1}(a, b)=\varphi_{1}(a, b) \psi_{1}\left(\varphi_{2}(a, b) \varphi_{1}(a, b)\right) \\
& =(a, f(a, b)) \psi_{1}((g(a, b), b)(a, f(a, b)) \\
& =(a, f(a, b))(g(a, b), h(g(a, b), f(a, b)) \\
& =(a, h(g(a, b), f(a, b))) .
\end{aligned}
$$

Similarly, we obtain $(\varphi * \psi)_{2}(a, b)=(k(g(a, b), f(a, b)), b)$. Hence $\varphi * \psi$ is determined by a single function: $X \times X \longrightarrow$ $X \times X$ with the rule

$(a, b)$

$$
\longrightarrow(k(g(a, b), f(a, b)), h(g(a, b), f(a, b))) .
$$

Let $S$ be a semigroup, $I$ and $\wedge$ nonempty sets, and $P$ a matrix indexed by $I$ and $\wedge$ with entries $p_{i, \lambda}$ taken from $S$. Then the Rees matrix semigroup ([7]) $M(S ; I ; \wedge ; P)$ is the set $I \times S \times \wedge$ together with the multiplication

$$
(i, s, \lambda)(j, t, \mu)=\left(i, s p_{\lambda, j} t, \mu\right) .
$$

In the next example we describe flows on Rees matrix semigroups.

Example 3. The Rees matrix semigroup $M(S ; I ; \wedge ; P)$ is regular since, for all elements $(i, a, \lambda)$ in this semigroup, there exist $(j, b, \mu)$ such that

$$
(i, a, \lambda)(j, b, \mu)(i, a, \lambda)=(i, a, \lambda)
$$

and

$$
(j, b, \mu)(i, a, \lambda)(j, b, \mu)=(j, b, \mu) .
$$

From (34), we get

$$
\begin{aligned}
a & =a p_{\lambda j} b p_{\mu i} a \longrightarrow 1=p_{\lambda j} b p_{\mu i} \longrightarrow b \\
& =\left(p_{\lambda j}\right)^{-1} a^{-1}\left(p_{\mu i}\right)^{-1},
\end{aligned}
$$

and from (35), we have

$$
\begin{aligned}
b & =b p_{\mu i} a p_{\lambda j} b \longrightarrow 1=p_{\mu i} a p_{\lambda j} b \longrightarrow b \\
& =\left(p_{\lambda j}\right)^{-1} a^{-1}\left(p_{\mu i}\right)^{-1} .
\end{aligned}
$$

So $V(i, a, \lambda)=\left\{(j, b, \mu): b=\left(p_{\lambda j}\right)^{-1} a^{-1}\left(p_{\mu i}\right)^{-1}\right\}$. Hence once we have selected $j$ and $\mu, b$ is uniquely determined.

For any element $e=(i, a, \lambda) \in M(S ; I ; \wedge ; P)$ is an idempotent if

$$
\begin{aligned}
(i, a, \lambda)(i, a, \lambda) & =(i, a, \lambda) \longrightarrow\left(i, a p_{\lambda i} a, \lambda\right) \\
& =(i, a, \lambda) \longrightarrow a=\left(p_{\lambda_{i}}\right)^{-1}
\end{aligned}
$$

So $E(M(S ; I ; \wedge ; P))=\left\{\left(i,\left(p_{\lambda i}\right)^{-1}, \lambda\right): i \in I, \lambda \in \wedge\right\}$. Therefore we can think of $E(M(S ; I ; \wedge ; P))$ as being identified (once we are given $P$ ) with $I \times \wedge$.
For each $(i, \mu) \in I \times \wedge$, a flow $\varphi \in \Phi(M(S ; I ; \wedge ; P))$ determines a pair of inverse elements $(i, a, \lambda),(j, b, \mu)$ (in which $\left.b=\left(p_{\lambda j}\right)^{-1} a^{-1}\left(p_{\mu i}\right)^{-1}\right)$ such that

$$
(i, a, \lambda),(j, b, \mu)=\left(i, a p_{\lambda j} b, \mu\right)=\left(i,\left(p_{\mu i}\right)^{-1}, \mu\right) .
$$

Once $i$ is determined, we have free choice for $a, \lambda, j$ and $\mu$ is determined, and so $b$ is determined. Hence flow is a function $\varphi: I \times \wedge \longrightarrow S \times \wedge \times I$. We can now think of a flow $\varphi$ as two functions:

$$
\begin{aligned}
& \delta_{\varphi}: I \times \wedge \longrightarrow S \quad(i, \mu) \longmapsto a \\
& \pi_{\varphi}: I \times \wedge \longrightarrow I \times \wedge \quad(i, \mu) \longmapsto(j, \lambda) .
\end{aligned}
$$

We determine the composition of functions that corresponds to the composition of flows. Take two flows $\varphi_{1}$ and $\varphi_{2}$ with corresponding functions

$$
\begin{gathered}
\delta_{1}, \delta_{2}^{\prime}: I \times \wedge \longrightarrow S \\
\text { and } \pi_{1}, \pi_{2}^{\prime}: I \times \wedge \longrightarrow I \times \wedge .
\end{gathered}
$$

We have

$$
\begin{aligned}
\left(\varphi_{1} * \varphi_{2}\right)(i, \mu) & =\varphi_{1}(i, \mu) \cdot \varphi_{2}\left(\tau \varphi_{1}(i, \mu)\right) \\
& =\varphi_{1}(i, \mu) \cdot \varphi_{2}\left(\pi_{1}(i, \mu)\right)
\end{aligned}
$$

Now $\varphi_{1}(i, \mu)=\left(\left(i, \delta_{1}(i, \mu), \lambda_{1}\right),\left(j_{1}, b_{1}, \mu\right)\right)$, where $\pi_{1}(i, \mu)=$ $\left(j, \lambda_{1}\right)$ and

$$
\begin{aligned}
\varphi_{2}\left(\pi_{1}(i, \mu)\right) & =\varphi_{2}\left(j_{1} \lambda_{1}\right) \\
& =\left(\left(j_{1}, \delta_{2}\left(j_{1}, \lambda_{1}\right), \lambda_{2}\right),\left(j_{2}, b_{2}, \lambda_{1}\right)\right),
\end{aligned}
$$

where $\pi_{2}\left(j_{1}, \lambda_{1}\right)=\left(j_{2}, \lambda_{2}\right)$. So

$$
\begin{aligned}
& \left(\varphi_{1} * \varphi_{2}\right)(i, \mu)=\left(\left(i, \delta_{1}(i, \mu), \lambda_{1}\right)\right. \\
& \left.\cdot\left(j_{1}, \delta_{2}\left(j_{1}, \lambda_{1}\right), \lambda_{2}\right),\left(j_{2}, b_{2}, \lambda_{1}\right)\left(j_{1}, b_{1}, \mu\right)\right) \\
& \quad=\left(\left(i, \delta_{1}(i, \mu) p_{\lambda_{1} j_{1}} \delta_{2}\left(j_{1}, \lambda_{1}\right), \lambda_{2}\right),\right. \\
& \left.\quad\left(j_{2}, b_{2} p_{\lambda_{1} j_{1}} b_{1}, \mu\right)\right) .
\end{aligned}
$$

The functions $\delta$ and $\pi$ corresponding to $\varphi_{1} * \varphi_{2}$ are

$$
\begin{aligned}
& \begin{aligned}
\delta(i, \mu) & =\delta_{1}(i, \mu) p_{\lambda_{1} j_{1}} \delta_{2}\left(j_{1}, \lambda_{1}\right) \\
& =\delta_{1}(i, \mu) p_{\lambda_{1} j_{1}} \delta_{2}\left(\pi_{1}(i, \mu)\right) \\
& =\delta_{1}(i, \mu) p_{\overline{\pi_{1}}(i, \mu)} \delta_{2}\left(\pi_{1}(i, \mu)\right)
\end{aligned} \\
& \left(\overline{\pi_{1}}(i, \mu)=\left(\lambda_{1}, j_{1}\right) \text { - switching order of components }\right) \\
& \pi(i, \mu)=\left(j_{2}, \lambda_{2}\right)=\pi_{2}\left(j_{1}, \lambda_{1}\right)=\pi_{2} \pi_{1}(i, \mu)
\end{aligned}
$$

Thus $\pi=\pi_{2} \circ \pi_{1}$, and so, for any flow monoid $\Phi(\mathbb{C})$ on any category $\mathbb{C}$, we get a monoid homomorphism $(X$ is the obj $\mathbb{C})$ )

$$
\pi: \Phi(\mathbb{C}) \longrightarrow \operatorname{End}(X)^{o p}
$$

such that elements $p_{\lambda_{1} j_{1}}$ occur to reflect rule for multiplication in an $H$-class of $M(S ; I ; \wedge ; P)$. 


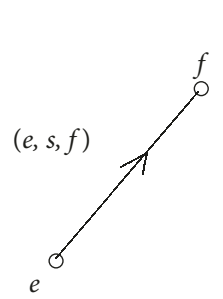

(a)

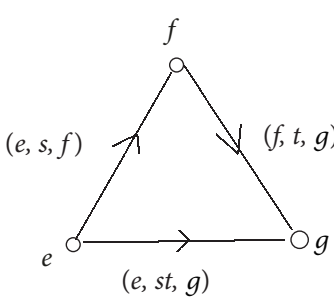

(b)

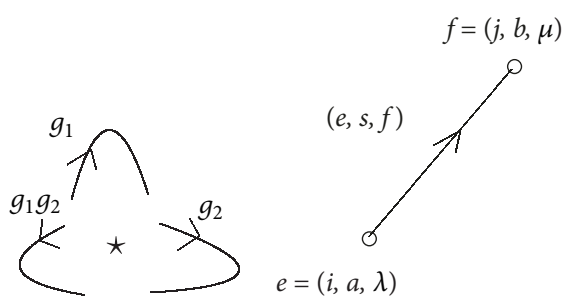

(d)

Figure 1: (a) An arrow in $\varrho(S)$. (b) Composition of arrows in $\varrho(S)$. (c) Category with one vertex. (d) Typical arrow in Rees matrix semigroup.

Let $S=M(H ; I ; \wedge ; P)$ be a Rees matrix group. Consider the subset $H_{i \lambda}=\{(i, h, \lambda): h \in H\}$ of $S$. In fact $H_{i \lambda}$ is a subgroup of $S$ which is isomorphic to $H$. Consider

$$
(i, h, \lambda)\left(i, h^{\prime}, \lambda\right)=\left(i, h p_{\lambda i} h^{\prime}, \lambda\right) \in H_{i \lambda}
$$

Thus we have the following lemma and result.

Lemma 4. Let $S$ be a group, and fix an element $p \in S$. Define a new binary operation $*_{p}$ on $S$ as follows: for all $a, b \in S$, $a *_{p} b=a p b \in S$. Then $\left(S, *_{p}\right)$ is a group isomorphic to $H$.

Corollary 5. $S=M(H ; I ; \wedge ; P)$ contains many copies of the group $H$, all isomorphic to $H$ one copy $H_{i \lambda}$ for each $(i, \lambda) \epsilon$ $I \times \wedge$.

\section{Flows on Cauchy Categories}

In this section we will investigate another category that can be built from a semigroup. No special conditions are required: any semigroup $S$ gives us its Cauchy category $\varrho(S)$.

The Cauchy category is defined as follows: the set of vertices (or objects) is $E(S)$. Given $e, f \in E(S)$ the set of arrows in $\varrho(S)$ from $e$ to $f$ is

$$
\varrho(e, f)=\{(e, s, f): e s=s=s f\} .
$$

as in Figure 1(a).

The identity arrow at $e \in E(S)$ is $(e, e, e)$ and the composition of arrows is shown in Figure 1(b).

$$
(e, s, f)(f, t, g)=(e, s t, g) \text {. }
$$

Lemma 6. In the Cauchy category $\varrho(S),(e, s, f) \in \rho(e, f)$ if and only if $s \in e S f$. Hence $\varrho(e, f)$ is always nonempty (so that $\varrho$ is a connected category).

Proof. Suppose that $(e, s, f) \in \varrho(e, f)$; then $e s=s=s f$. Hence $s=e s f \Longrightarrow s \in e S f$, where $s \in S$.

Conversely, if $s \in e S f$, then $s=e$ af where $a \in S$. We want to show that $e s=s=s f$. Since

$$
\begin{aligned}
& e s=e(e a f)=e^{2} a f=e a f=s \\
& \quad(e \text { is an idempotent }) ; \\
& s f=(e a f) f=e a f^{2}=e a f=s
\end{aligned}
$$

( $f$ is an idempotent).
Hence $e s=s=s f$; therefore $(e, s, f) \in \varrho(e, f)$.

Since eSf $\neq \phi$, and if $e=f$, the identity arrow at $e \in E(S)$ is $(e, e, e) \in \varrho(e, f)$ such that $e e=e^{2}=e$. Therefore $\varrho(e, f)$ is always nonempty.

If we thought of $\varrho$ as a category with one vertex, then we have the following. If $G$ is a group then $\varrho(G)$ is equal to $G$. We have one object (vertex *) which is not an element of $G$ and one arrow for each element of $G$, with the arrow $1 \in G$ being the identity arrow at $*$. Hence we have one arrow for each element of $G$; i.e., $\varrho(G)=G$. See Figure $1($ c).

Lemma 7. Let $M$ be a monoid with identity 1 ; then $\{(1, m, 1)$ : $m \in M\}$ is a submonoid of $\varrho(M)$ and isomorphic to $M$. Hence $M$ is a submonoid of $\Phi(\varrho(M))$.

Proof. Let $\bar{M}=\{(1, m, 1): m \in M\}$ be a submonoid of $\varrho(M)$. It is easy to check that the operation is associative since associativity will hold automatically in a category. Also $(1,1,1) \in \bar{M}$ is the identity arrow. To show that $\bar{M} \simeq M$, suppose $f: M \longrightarrow \bar{M}$ defined by $f(m)=(1, m, 1)$ again it is easy to check that $f$ is one-one correspondence and it is a morphism. In order to prove that $M$ is a submonoid of $\Phi(\varrho(M))$, given $m \in M$, define a flow $\varphi_{m}$ on $\varrho(M)$ as follows:

$$
\begin{aligned}
\varphi_{m}(1) & =(1, m, 1) \\
\text { and } \varphi_{m}(e) & =(e, e, e), \quad e \neq 1 .
\end{aligned}
$$

Then $\left\{\varphi_{m}: m \in M\right\}$ is isomorphic to $M$; this gives us a copy of $M$ inside $\Phi(\varrho(M))$. Hence $M$ is a submonoid of $\Phi(\varrho(M))$.

In the following we describe the Cauchy categories of some small semigroups given in [7].

(i) Cauchy category of a rectangular band $S$ : we write $S=$ $A \times B$ using $a b a=a$ for all $a, b \in S$. If $(e, s, f) \in \varrho(e, f)$ then $s \in e S f:$ so $s=$ et $f$ for some $t \in S$ and $e, f \in E(S)$. But it follows that

$$
\begin{aligned}
s & =e t f=e(e t f) f \\
& =e(\text { etf }) f e f \quad(\text { since } f e f=f) \\
& =e(\text { etf }) e f=e^{2} f=e f
\end{aligned}
$$

The set of vertices (or objects) of $\varrho(S)$ is $E(S)=S$, and arrow from idempotent $(a, b)$ to idempotent $(c, d)$ is defined 


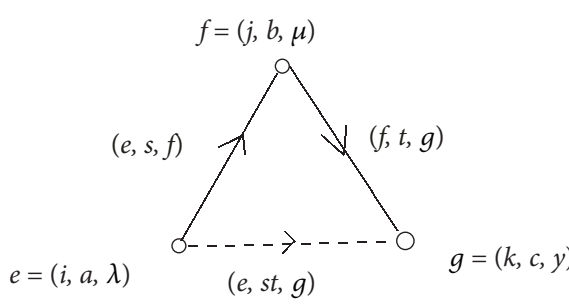

(a)

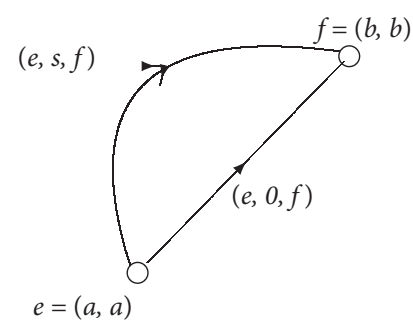

(b)

Figure 2: (a) Composition of arrows in Rees matrix semigroup. (b) Arrows in Brandt semigroup.

by element $(x, y) \in S$ such that $(\underbrace{(a, b)}_{e}, \underbrace{(x, y)}_{s}, \underbrace{(c, d)}_{f}) \in \varrho(S)$ where es $=(a, b)(x, y)=(a, y)=(x, y)$ so $x=a$ and $s f=$ $(x, y)(c, d)=(x, d)=(x, y)$ so $y=d$. Hence $(e, e f, f)$ is the only arrow connecting $e, f$.

If we have in the Cauchy category of a regular semigroup $S$ which has exactly one arrow from any two idempotents vertices, assume that et $f=$ ef for all $e, f$ in the set of idempotents $E(S)$ and $t \in S$. If we put $t=f e$ then et $f=$ efef $=(e f)^{2}=e f \in E(S)$. Hence $E(S)$ is a subsemigroup of $S$.

Now since $S$ is regular let $a \in S$ and $b \in V(a)$; then $a b, b a \in$ $E(S), e=a b, f=b a$ and $t=a$; we want to prove that every element of $S$ is an idempotent. Since et $f \in S$ is an arbitrary element of $S$,

$$
\begin{aligned}
(\text { etf })^{2} & =(\text { etf })(\text { etf })=a b \underbrace{a b} a \underbrace{a b} a b a=a b a b a \\
& =a b a=a ;
\end{aligned}
$$

and

$$
\text { etf }=a b a b a=a b a=a
$$

Hence every element of $S$ is an idempotent; therefore $S$ is a band; our assumption on $S$ tells us that for all $a, b, c \in S, a b c=$ $a c$, if $c=a$, then $a b a=a^{2}=a$. Therefore $S$ is a rectangular band.

We prove the coming result.

Proposition 8. Let $S$ be a regular semigroup. Then in the Cauchy category $\varrho(S)$ we have exactly one arrow from e to $f$ (for all e, $f \in E(S)$ ) if and only if $S$ is a rectangular band.

Take the semigroup $S$ with $E(S)=\varphi$ (for example, infinite monogenic semigroup). Define $S^{0}=S \cup\{0\} ; 0 s=s 0=0$, and $00=0$ for all $s \in S$; then $E\left(S^{0}\right)=\{0\}$ and $S^{0}$ satisfies our assumption: et $f=e f$. Since we must have $e=0=f$, $S^{0}$ is not regular ( 0 is the only regular element). Can we find a semigroup $S$ that does not have a zero, is not regular, and satisfies for all $e, f \in E(S), t \in S$ such that et $f=e f$ ? Consider $T=\left\{x, x^{2}\right\}, x^{3}=x^{2}$ (which is a monogenic subsemigroup) is not a band since $x \neq x^{2}$ and not regular since $x$ is not a regular element, but satisfies $x y z=x z$ for all $x, y, z$ since

\begin{tabular}{ccc}
\hline$x$ & $y$ & $z$ \\
\hline$x$ & $x$ & $x$ \\
\hline$x$ & $x$ & $x^{2}$ \\
\hline$x$ & $x^{2}$ & $x^{2}$ \\
\hline$x^{2}$ & $x$ & $x$ \\
\hline$x^{2}$ & $x$ & $x^{2}$ \\
\hline$x^{2}$ & $x^{2}$ & $x$ \\
\hline$x$ & $x^{2}$ & $x$ \\
\hline$x^{2}$ & $x^{2}$ & $x^{2}$
\end{tabular}$=x^{2}$.

Define $S=\mathbb{N} \times T$ with multiplication $(m, y)(n, z)=(n, y z)$, which is a semigroup such that no element acts as 0 (if $(n, z) \epsilon$ $S$ behave like a zero then $z=x^{2} .(n, z)(m, y)=(m, z) \neq(n, z)$ if $m \neq n$ ) moreover $(n, x)$ is not regular for any $n \in \mathbb{N}$ : we compute $(n, x)(m, y)(n, x)=\left(n, x^{2}\right) \neq(n, x)$ for all $y$. But $S$ satisfies the low etf $=$ ef since $\left(m_{1}, y_{1}\right)\left(m_{2}, y_{2}\right)\left(m_{3}, y_{3}\right)=$ $\left(m_{3}, y_{1} y_{2} y_{3}\right)=\left(m_{3}, y_{1} y_{3}\right)=\left(m_{1} y_{1}\right)\left(m_{3}, y_{3}\right)$ (remember that $y_{1} y_{2} y_{3}=y_{1} y_{3}$ in $\left.T\right)$. Hence if $T$ satisfies $a b c=a c$ as a low, then $S$ satisfies same low. As a semigroup, if $T$ is a rectangular band, then so is $S$.

(i) We describe Cauchy categories $\varrho(S)$ where $S$ is completely simple semigroup which is the Rees matrix semigroup $S=M[I, \wedge, P, H]$ where $(i, a, \lambda)(j, b, \mu)=$ $\left(i, a p_{\lambda j} b, \mu\right)$. The vertex set is $E(S)=\{e: e=$ $\left.(i, a, \lambda), a=\left(p_{\lambda i}\right)^{-1}\right\}$. The arrows between the vertices $e=(i, a, \lambda)$ and $f=(j, b, \mu)$ are $l=(e, s, f)$ where $s=(i, c, \mu)$ such that $e s=s, s f=s$. See Figure $1(\mathrm{~d})$.

Check the composition of arrows: So let $e=$ $(i, a, \lambda), a=p_{\lambda i}^{-1}, f=(j, b, \mu), b=p_{\mu j}^{-1}$ and $g=$ $(k, c, \gamma), c=p_{\gamma k}^{-1}$ as drawn in Figure 2(a). If $s=(i, d, \mu)$ and $t=(j, h, \gamma)$, then

$$
\begin{aligned}
e(s t) & =(i, a, \lambda)((i, d, \mu)(j, h, \gamma)) \\
& =(i, a, \lambda)\left(i, d p_{\mu j} h, \gamma\right)=\left(i, a p_{\lambda i} d p_{\mu j} h, \gamma\right) \\
& =\left(i, d p_{\mu j} h, \gamma\right)=s t
\end{aligned}
$$

and

$$
\begin{aligned}
(s t) g & =\left(i, d p_{\mu j} h, \gamma\right)\left(k, p_{\gamma k}^{-1}, \gamma\right)=\left(i, d p_{\mu j} h p_{\gamma k} p_{\gamma k}^{-1}, \gamma\right) \\
& =s t .
\end{aligned}
$$

Identity at $e \in E(S)$ is $(e, e, e)$. 


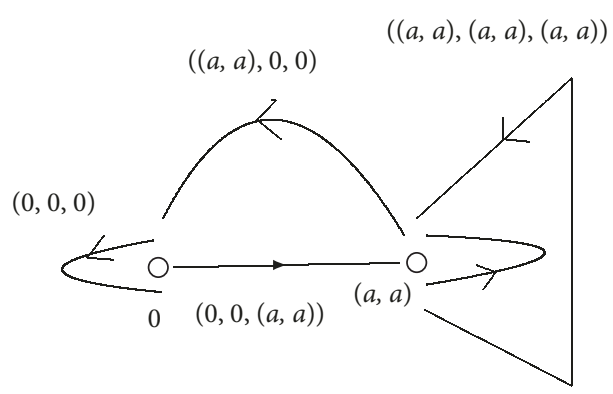

Figure 3: Cauchy category $\varrho\left(B_{2}\right)$.

(ii) We describe Cauchy categories $\varrho(S)$ where $S$ is a Brandt semigroup $B_{2}$. The general construction of Brandt semigroups proceeds as follows. Let $X$ be a finite set with $n$ elements. The Brandt semigroup $B_{n}$ is the set $B_{n}=(X \times X) \cup\{0\}$ with multiplication given by

$$
(a, b)(c, d)= \begin{cases}(a, d) & \text { if } b=c \\ 0 & \text { otherwise }\end{cases}
$$

so $B_{n}$ has $n^{2}+1$ elements, and $n+1$ idempotents:

$$
E\left(B_{n}\right)=\{(a, a): a \in X\} \cup\{0\} .
$$

Arrows from idempotent $e=(a, a)$ to idempotent $f=(b, b)$ are a triple $(e, s, f)$ such that $S=(x, y)$ such that $(e s=s=s f)$, $e s=(a, a)(x, y)=(a, y)$ if $a=x$ and $s f=(x, y)(b, b)=(x, b)$ if $b=y$. So if $e, f \neq 0$ then $e=(a, a), f=(b, b)$, then $(a, b) \in \varrho(S)$ but if $a \neq b$, then ef $=0$, and so we get two arrows from $e$ to $f$ as shown in Figure 2(b).

$$
\begin{gathered}
(e, 0, f) \\
\text { and }(e,(a, b), f)
\end{gathered}
$$

Moreover, $0 \in E(S)$ and 0 is a vertex of $\varrho(S)$. Then $0 \cdot s=0$ so $s=0$ and then $s f=0 f=0$.

Now $B_{n}$ has $n+1$ idempotents. If $n+1 \geq 3$ there are at least two nonzero idempotents and $e, f \neq 0$; then $\varrho(S)$ has two arrows. If $n=1, B_{1}$ has only two elements $B_{1}=\{(a, a), 0\}$ which is certainly a band; but since $(a, a) 0(a, a)=0 \neq(a, a)$ it is not a rectangular band. See Figure 3.

Our task here is to describe the flow monoid on a Cauchy category. We describe it in terms of functions defined on $E(S)$. A flow $\phi$ on $\varrho(S)$ assigns to each $e \in E(S)$ an arrow $(e, s, f)$ where $e, f \in E(S)$ and $s \in e S f$. So we can first select the function $\pi_{\phi}: e \longmapsto f$, and then having chosen $\pi_{\phi}$ we have a second function $\delta_{\phi}$ say, with $\delta_{\phi}: E(S) \longrightarrow S$ satisfying $\delta_{\phi}(e) \in e S \pi_{\phi}(e)$ for all $e$. The pair $\left(\pi_{\phi}, \delta_{\phi}\right)$ then determines the flow $\phi$. If $\phi_{1}$ and $\phi_{2}$ are two flows determined by the pairs $\left(\pi_{1}, \delta_{1}\right)$ and $\left(\pi_{2}, \delta_{2}\right)$, then we compute the composition of flows $\phi_{1} * \phi_{2}$ as

$$
\begin{aligned}
& \left(\phi_{1} * \phi_{2}\right) x=\phi_{1}(x) \cdot \phi_{2}\left(\pi_{1}(x)\right) \\
& =\left(x, \delta_{1}(x), \pi_{1}(x)\right)\left(\pi_{1}(x), \delta_{2}\left(\pi_{1}(x)\right), \pi_{2}\left(\pi_{1}(x)\right)\right) \\
& =\left(x, \delta_{1}(x) \delta_{2}\left(\pi_{1}(x)\right), \pi_{2}\left(\pi_{1}(x)\right) .\right.
\end{aligned}
$$

For future work from the point of semigroup theory, it is quite natural to consider partial flows that are only defined on a subset of $X$. This is similar idea of considering the partial transformation semigroup $\wp_{X}$ of a set $X$ instead of the full transformation semigroup $\mathfrak{\Im}_{X}$. Partial flow then is a function from a subset $U \subseteq X$ assigning to each $u \in U$ an arrow $\phi(u)$ starting at $u$; composition of $\phi$ defined on $U$ and $\psi$ defined on $V$ is defined on the subset $W=\{u \in U: \phi(u)$ ends at an element of $V\}$ and then $(\phi * \psi)(u)=\phi(u) \cdot \psi(\tau \phi(u))$; we need to allow the empty flow which acts as a zero; $\Phi(C)$ comes equipped with a monoid homomorphism $\pi(\phi): \Phi(C) \longrightarrow$ $\wp_{X}$ (partial transformation semigroup) such that $\pi(\phi): u \longmapsto$ $\tau(\phi(u))$. We will try to set up the theory of partial flows on category, generalizing [1, Sections 2 and 4]. Another future work for flows monoid is to present a Gröbner-Shirshov basis for each example in this paper. We may refer to [9-13] for more details.

\section{Data Availability}

The [flows monoid concept] used to support the findings of this study has been deposited in "Gilbert ND. Flows on Regular Semigroups. Applied Categorical Structures 2003. (11) 147-155. DOI 10.1007/s00233-008-9065-5 https://rdcu.be/ bwQNG" which is included within the reference of the article. The [Cauchy Categories concept] used to support the findings of this study has been deposited in "Costa, A. \& Steinberg, B. Semigroup Forum (2015) 91: 543. https://doiorg.sdl.idm.oclc.org/10.1007/s00233-014-9657-1" within the reference of this article. The unpublished manuscript " $S$. U. Chase, On representations of small categories and some constructions in algebra and combinatorics. Preprint (1977)" supports the findings of this study which are available from the corresponding author upon request.

\section{Conflicts of Interest}

The author declares that they have no conflicts of interest.

\section{References}

[1] N. D. Gilbert, "Flows on regular semigroups," Applied Categorical Structures. A Journal Devoted to Applications of Categorical Methods in Algebra, Analysis, Order, Topology and Computer Science, vol. 11, no. 2, pp. 147-155, 2003.

[2] F. Borceux, Handbook of Categorical Algebra. 1. Encyclopedia of Mathematics and its Applications, vol. 50 of Basic Category Theory, Cambridge University Press, Cambridge, 1994.

[3] F. Borceux, Handbook of Categorical Algebra. 2. Encyclopedia of Mathematics and its Applications, vol. 51 of Categories and Structures, Cambridge University Press, Cambridge, 1994.

[4] S. Mac Lane, "Categories for the working mathematician," in Graduate Texts in Mathematics, vol. 5, Springer, New York, NY, USA, 2nd edition, 1998.

[5] P. J. Higgins, "Notes on categories and groupoids," Van Nostrand Reinhold Mathematical Studies, vol. 32, 1971.

[6] A. Costa and B. Steinberg, "The Schützenberger category of a semigroup," Semigroup Forum, vol. 91, no. 3, pp. 543-559, 2015. 
[7] J. M. Howie, Fundamentals of Semigroup Theory, London Mathematical Society Monographs - Oxford University Press, 1997.

[8] M. V. Lawson, Inverse Semigroups: The Theory of Partial Symmetries, World Scientific Publishing Co., 1998.

[9] F. r. Ates, E. G. Karpuz, C. Kocapinar, and A. S. Cevik, "GröbnerShirshov bases of some monoids," Discrete Mathematics, vol.311, no. 12, pp. 1064-1071, 2011.

[10] F. r. Ates, A. S. Cevik, and E. G. Karpuz, "Gröbner-Shirshov basis for the singular part of the Brauer semigroup," Turkish Journal of Mathematics, vol. 42, no. 3, pp. 1338-1347, 2018.

[11] E. G. Karpuz, "Gröbner-Shirshov bases of some semigroup constructions," Algebra Colloquium, vol. 22, no. 1, pp. 35-46, 2015.

[12] E. G. Karpuz, F. Ates, and A. S. Cevik, "Gröbner-Shirshov bases of some Weyl groups," Rocky Mountain Journal of Mathematics, vol. 45, no. 4, pp. 1165-1175, 2015.

[13] C. Kocapinar, E. G. Karpuz, F. Ates, and A. S. Cevik, "GröbnerShirshov bases of the generalized Bruck-Reilly *-extension," Algebra Colloquium, vol. 19, pp. 813-820, 2012. 


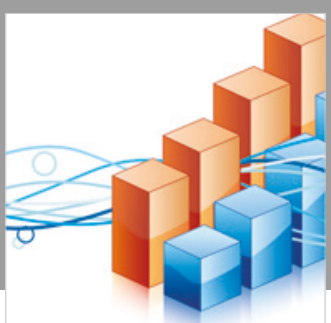

Advances in

Operations Research

\section{-n-m}
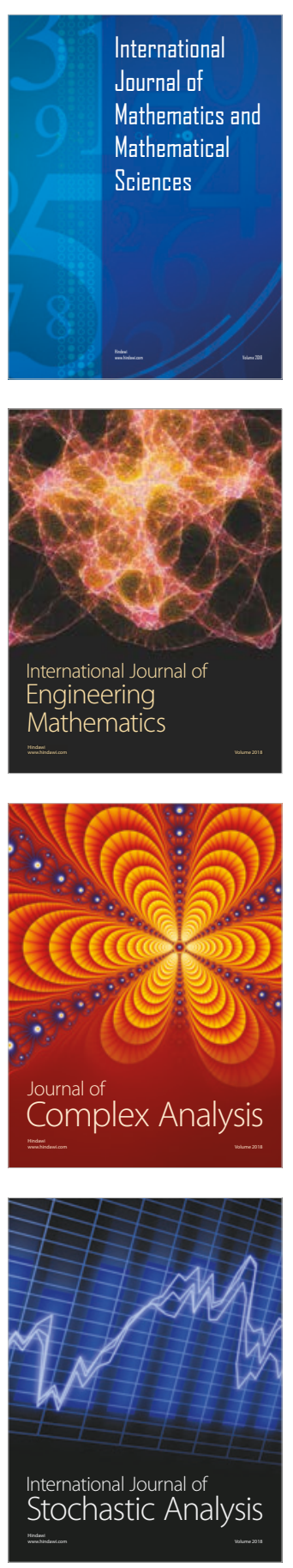
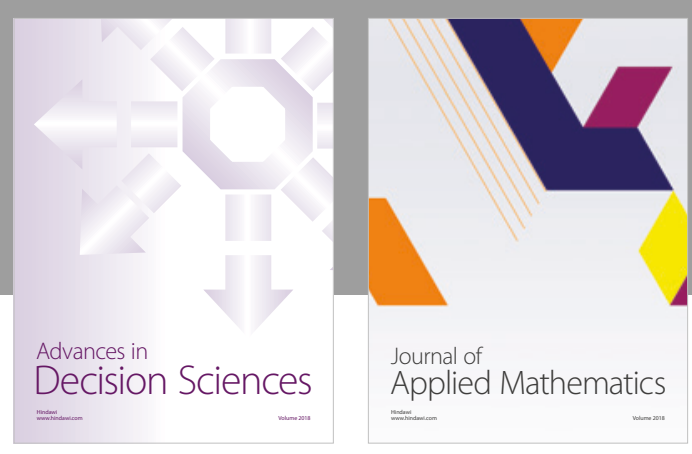

Journal of

Applied Mathematics
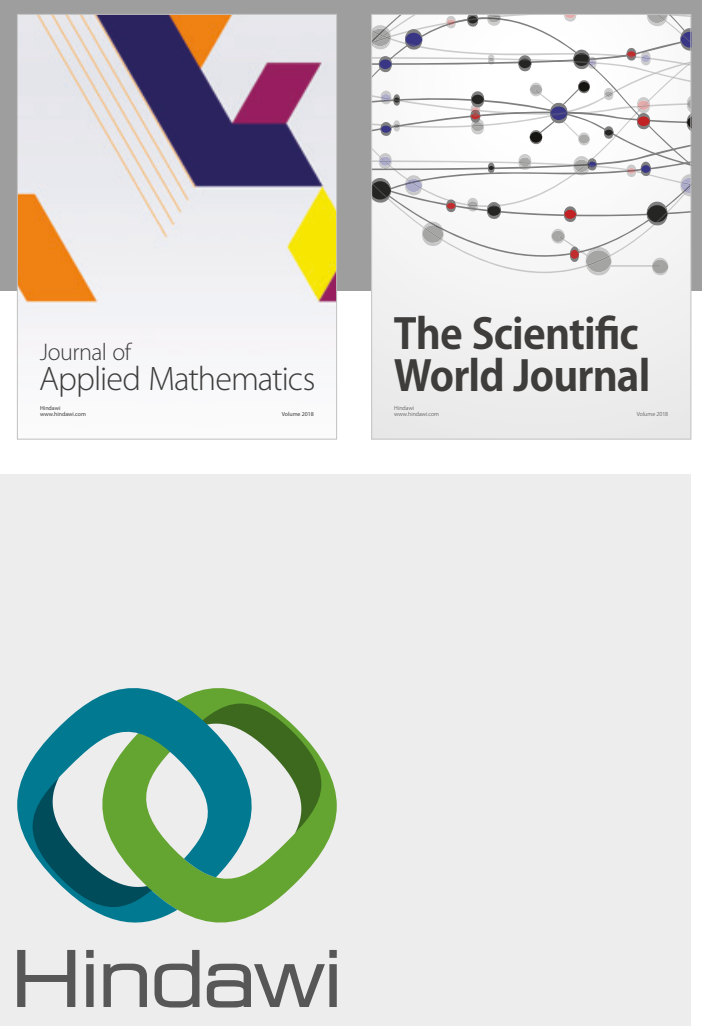

Submit your manuscripts at

www.hindawi.com

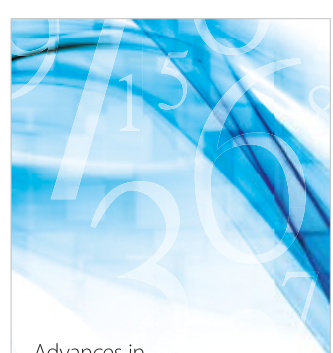

Advances in
Numerical Analysis
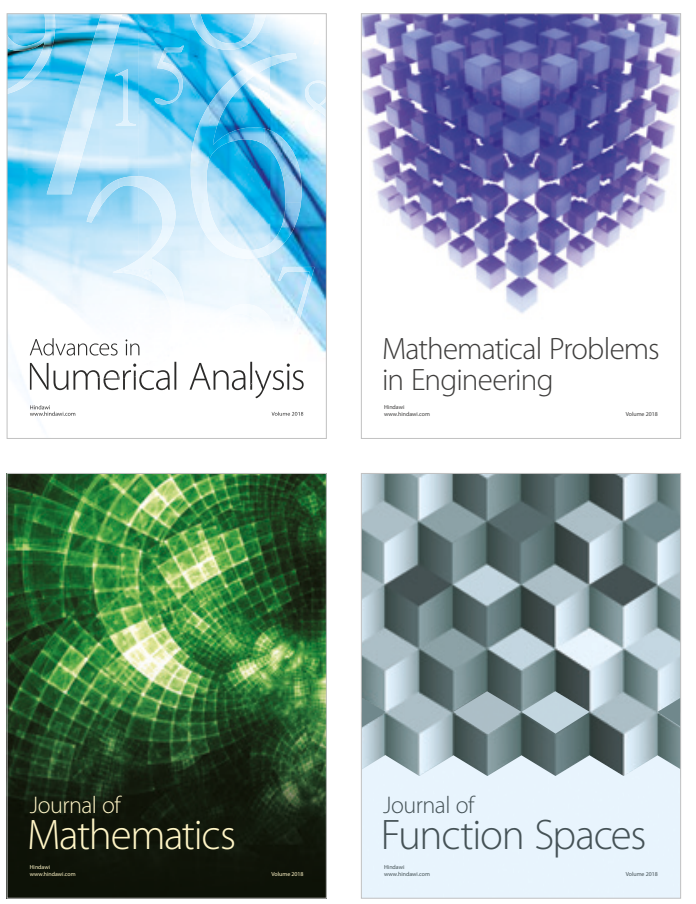

Mathematical Problems in Engineering

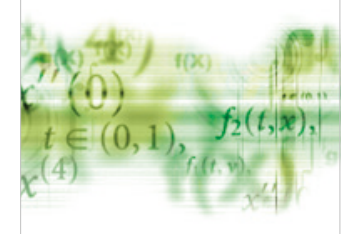

International Journal of

Differential Equations

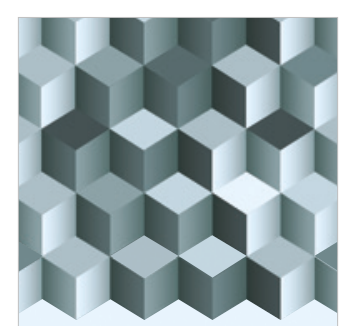

Journal of

Function Spaces

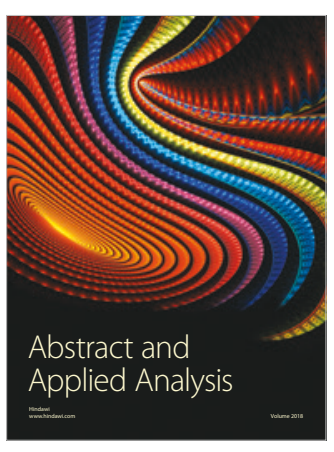

The Scientific

World Journal

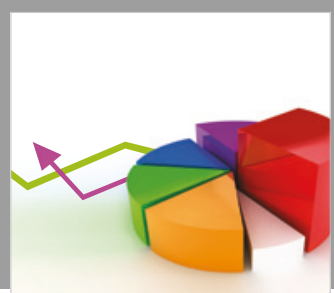

Journal of

Probability and Statistics
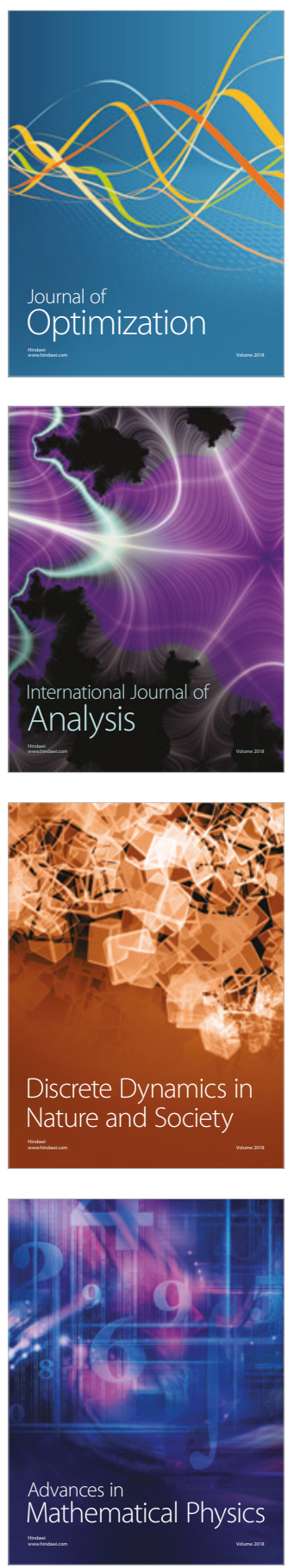